\title{
High-throughput phenotyping reveals differential transpiration behavior within the banana wild relatives highlighting diversity in drought tolerance
}

\author{
David Eyland ${ }^{1}$, Nathalie Luchaire ${ }^{2}$, Llorenç Cabrera-Bosquet ${ }^{3}$, Boris Parent ${ }^{3}$, Steven \\ Janssens $^{4}$, Rony Swennen ${ }^{1}$, Claude Welcker ${ }^{5}$, Francois Tardieu ${ }^{5}$, and sebastien carpentier ${ }^{1}$ \\ ${ }^{1}$ KU Leuven \\ ${ }^{2}$ INRAE \\ ${ }^{3}$ INRA-LEPSE \\ ${ }^{4}$ Meise Botanical Garden \\ ${ }^{5}$ INRA
}

August 2, 2021

\begin{abstract}
Crop wild relatives, the closely related species of crops, may harbor potentially important sources of new allelic diversity for (a)biotic tolerance or resistance. However, to date wild diversity is only poorly characterized and evaluated. Banana has a large wild diversity but only a narrow proportion is currently used in breeding programs. The main objective of this work was to evaluate genotype-dependent transpiration responses in relation to the environment. By applying continuous high-throughput phenotyping, we were able to construct genotype-specific transpiration response models in relation to light, VPD and soil water potential. We characterized and evaluated 6 (sub)species and discerned four phenotypic clusters. Significant differences were observed in leaf area, cumulative transpiration and transpiration efficiency. We confirmed a general stomatal-driven 'isohydric' drought avoidance behavior, but discovered genotypic differences in the onset and intensity of stomatal closure. We pinpointed crucial genotype specific environmental conditions when drought avoidance mechanisms were initiated and when stress kicked in. Differences between (sub)species were more pronounced under certain environmental conditions, illustrating the need for high-throughput dynamic phenotyping, modelling and validation. We conclude that the banana wild relatives contain useful drought tolerance traits, emphasizing the importance of their conservation and potential for use in breeding programs.
\end{abstract}

\section{Hosted file}

Eyland_EPPN_version4.docx available at https://authorea.com/users/428661/articles/532472high-throughput-phenotyping-reveals-differential-transpiration-behavior-within-thebanana-wild-relatives-highlighting-diversity-in-drought-tolerance 